\title{
Soft diffraction within the QCD color dipole picture
}

\author{
G. M. Peccini, ${ }^{*}$ F. Kopp $\odot,^{\dagger}$ and M. V. T. Machado $\odot^{\ddagger}$ \\ High Energy Physics Phenomenology Group, GFPAE. Institute of Physics, Federal University of Rio \\ Grande do Sul (UFRGS) Postal Code 15051, CEP 91501-970, Porto Alegre, RS, Brazil \\ D. A. Fagundes $\oplus^{\S}$ \\ Department of Exact Sciences and Education, CEE. Federal University of Santa Catarina \\ (UFSC)_Blumenau Campus, 89065-300 Blumenau, SC, Brazil
}

(Received 11 February 2020; accepted 13 April 2020; published 29 April 2020)

\begin{abstract}
In this work we consider the QCD parton saturation models to describe soft interactions at the high energy limit. The total and elastic cross sections, as well as the elastic slope parameter, are obtained for proton-proton and pion-proton collisions and compared to recent experimental results. The analyses are done within the color dipole formalism, taking into account saturation models which have been tested against deep inelastic scattering data. The main point is that the matching between soft and hard interaction occurs in the saturation region which can be described by high density QCD approaches. Discussion is performed on the main theoretical uncertainties associated with calculations.
\end{abstract}

DOI: 10.1103/PhysRevD.101.074042

\section{INTRODUCTION}

Describing soft processes using the QCD degrees of freedom is a quite difficult task as they are dominated by long distance (nonperturbative) physics. It has been shown that soft observables, as the total and elastic cross sections, depend on the transition region between the high parton density system (saturation domain) and perturbative QCD region [1-3]. The parton saturation phenomenon [4-6] is a well-established property of high energy systems and gives a high quality description of inclusive and exclusive deep inelastic scattering (DIS) data. As evidence for the successfulness of such approach we quote the description of the light meson photoproduction cross section [7-12] and diffractive DIS (DDIS) $[13,14]$. Both are hard processes, in which an important contribution to the cross section comes from the kinematic region in the vicinity of the saturation momentum, $Q_{s}$. This dimensional scale increases in the high energy region. A well-known formalism which is intuitive and where saturation physics can be easily implemented is the QCD color dipole picture. A seminal work on this approach is Ref. [15] where the

\footnotetext{
*guilherme.peccini@ufrgs.br

fabio.kopp@ufrgs.br

*magnus@if.ufrgs.br

§aniel.fagundes@ufsc.br
}

Published by the American Physical Society under the terms of the Creative Commons Attribution 4.0 International license. Further distribution of this work must maintain attribution to the author(s) and the published article's title, journal citation, and DOI. Funded by SCOAP. unitarity corrections to the proton structure function at small $x$ were derived and predictions are done for DDIS and nuclear shadowing. There it was demonstrated that the factorization of the photon-induced cross sections between the Fock states wave functions of photon and multiparticle dipole cross section provides clear identification of the partial waves of the dipole cross section as an object of the $s$-channel unitarization. Moreover, applications to the soft hadronic scattering within the same formalism have been done in [16]. It is expected [1] that soft processes measured, for instance, at the Large Hadron Collider (LHC) in hadronhadron collisions probe distances at about $r \sim 1 / Q_{s} \ll R_{h}$, with $R_{h}$ being the hadron radius. In this context, hadron scattering at the LHC could be described by color dipoles as the correct degrees of freedom even at large transverse distances. Moreover, it has been shown that the cross sections for soft hadron-hadron collisions within saturation approaches satisfy the Froissart-Martin bound [2,3]. In addition, the role played by the unitarized hard Pomeron contribution to the soft observables has also been carefully discussed in Refs. $[17,18]$. The relationship and equivalence between the Balitsky-Fadin-Kuraev-Lipatov (BFKL) and dipole equation kernels are investigated by means of explicit calculations in light-cone perturbation theory. A dipole equation, equivalent to the usual equation for interactions between four Reggeized gluons, is given in the large $N_{c}$ limit. The leading trajectory of the four-gluon system is bounded by $2 \alpha_{I P}-1$ with $\alpha_{I P}$ being the BFKL Pomeron intercept. [19]

An important property of the saturation formalism is the geometric scaling phenomenon [20], which means that the scattering amplitude and corresponding cross sections can 
scale on the dimensionless scale $\tau=\mu^{2} / Q_{s}^{2}$, where $\mu^{2}$ is the typical hard scale in the scattering process. For instance, $\mu^{2}=Q^{2}$ is the photon virtuality in DIS (i.e., the nucleon structure functions $F_{2}, F_{L}$ ) and deeply virtual Compton scattering (DVCS) processes or $\mu^{2}=\left(Q^{2}+M_{V}^{2}\right)$ in the case of exclusive electroproduction $\left(Q^{2} \neq 0\right)$ and photoproduction $\left(Q^{2}=0\right)$ of vector mesons of masses $M_{V}$. The treatment of vector meson production (including production of their excited states) within the color dipole picture in terms of the scanning radius, $r_{S}$, was first addressed in Ref. [21] with the identification of the relevant hard scale $\left(Q^{2}+M_{V}^{2}\right)$ (for a comprehensive and pedagogical review about vector mesons we quote Ref. [22], where experimental results are summarized and theoretical formalisms are compared with emphasis on the BFKL color dipole and $k_{t}$-factorization approaches). Deviations from geometric scaling are also known when the system is far from the saturation domain. Geometric scaling is an intrinsic property of nonlinear QCD evolution equations [4-6] in the asymptotic energy regime, $\sqrt{s} \rightarrow \infty$. This scaling property has been used in recent years to construct phenomenological models for the QCD dynamics at high energies. A very intuitive picture of inclusive or exclusive DIS processes is the color dipole picture [23-26]. In that picture the deep inelastic scattering process can be seen as a succession in time of three factorizable subprocesses: (i) the photon fluctuates in a quark-antiquark pair with transverse separation $r \sim 1 / Q$ long after the interaction, (ii) this color dipole interacts with the nucleon target, and (iii) the quark pair is projected into the considered final state. The nucleon structure function is related to the $\gamma^{*} p$ cross section as $F_{2}\left(x, Q^{2}\right)=\frac{Q^{2}}{4 \pi^{2} \alpha_{e m}} \sigma_{\text {tot }}^{\gamma^{*} p}$. The latter is the overlap of the dipole cross section on the transverse and longitudinal photon wave functions. The interaction is then factorized in the simple formulation [23-26],

$$
\begin{aligned}
\sigma_{\mathrm{tot}}^{\gamma^{*} p}\left(W_{\gamma p}, Q^{2}\right)= & \int d z d^{2} \boldsymbol{r}\left(\left|\Psi_{T}(z, \boldsymbol{r})\right|^{2}+\left|\Psi_{L}(z, \boldsymbol{r})\right|^{2}\right) \\
& \times \sigma_{\mathrm{dip}}(\tilde{x}, \boldsymbol{r}),
\end{aligned}
$$

where $z$ is the longitudinal momentum fraction of the quark in the color dipole, $\tilde{x}=\frac{Q^{2}+m_{q}^{2}}{W_{\gamma p}^{2}+Q^{2}}$ is equivalent to the Bjorken variable and provides an interpolation for the $Q^{2} \rightarrow 0$ limit. The mass of the quark of flavor $f$ is labeled as $m_{f}$. The photon wave functions $\Psi_{T, L}\left(r, z ; Q^{2}\right)$ are determined from light-cone perturbation theory and the dipole hadron cross section $\sigma_{\text {dip }}(x, r)=2 \int d^{2} b N_{\text {dip }}(x, r, b)$ contains all the information about the target and the strong interaction physics (including the impact-parameter $b$ dependence). As an example, the celebrated Golec-Biernat Wusthoff (GBW) parametrization $[14,27]$ takes the eikonal-like form,

$$
\begin{gathered}
\sigma_{\mathrm{dip}}\left(x, \boldsymbol{r}^{2}\right)=\sigma_{0}\left[1-\exp \left(-\frac{\boldsymbol{r}^{2} Q_{s}^{2}}{4}\right)^{\gamma_{s}}\right], \\
Q_{s}^{2}(x)=\left(\frac{x_{0}}{\tilde{x}}\right)^{\lambda} \mathrm{GeV}^{2},
\end{gathered}
$$

where $Q_{s}$ is the saturation scale. The parameters are obtained from the fit to the HERA data producing $\sigma_{0}=27.43 \mathrm{mb}, \lambda=0.248$, and $x_{0}=0.40 \times 10^{-4}$ for a five-flavor analysis (See Ref. [28] for an updated fitting procedure). Here, additional parameters are the effective light quark mass, $m_{f}=0.14 \mathrm{GeV}$, which plays the role of a regulator at the photoproduction limit. The charm (bottom) mass is set to be $m_{c}=1.4(4.6) \mathrm{GeV}$. The GBW parametrization presents a geometric scaling form, $\sigma_{\text {dip }} \propto f\left(\boldsymbol{r}^{2} Q_{\text {sat }}^{2}\right)$. For small dipoles $\boldsymbol{r}^{2} \leq 1 / Q_{\text {sat }}^{2}$ it can be approximated by $\sigma_{\text {dip }} \simeq \sigma_{0}\left(\boldsymbol{r}^{2} Q_{\text {sat }}^{2} / 4\right)$, where the effective anomalous dimension is equal to one, $\gamma_{s}=1$.

The advent of the LHC opened a new window for the studies on diffraction, elastic and inelastic scattering, as they are not strongly contaminated by nondiffractive events. This is translated in the Regge theory language by saying that the scattering amplitude is completely determined by a Pomeron exchange. The current measurements on these soft observables at the LHC in proton-proton collisions span a wide range energies from $100 \mathrm{GeV}$ including the very recent LHC data at 2.76, 7.0, 8.0, and $13 \mathrm{TeV}$ [29-37]. In the context of saturation physics the soft Pomeron may be understood as a unitarized perturbation Pomeron [38]. It can be shown that the trajectory of the soft Pomeron could emerge as a result of the interplay between perturbative physics of the hard Pomeron and the confining properties of the QCD vacuum. Specifically, local unitarization in the impact-parameter plane can lead to a reasonable description of intercept and the slope of the soft Pomeron [38]. In the present work, we investigate the soft observable in the small- $t$ regime within the color dipole picture and parton saturation approaches. Of course, some words of reservation are needed here. We are aware that the saturation scale, in general, is relatively small and its role in perturbative QCD (pQCD) is highly debatable. The issue of the extension of the color dipole language from the hard BFKL pQCD region of small dipoles to the soft Pomeron at hadronic scales remains an open one (we quote Ref. [39] and references therein a careful discussion of this problem). The paper is organized as follows. In the next section we summarize the theoretical information to compute the cross section for hadron-hadron collisions in two color dipole approaches. First, we consider the asymptotic cross section following Ref. [3], where the $p p$ cross section is assumed to be dominated by two-gluon production in the final state, $p p \rightarrow g g+X$. There, the main ingredients are the gluon distribution of the projectile and the partonic cross section associated to the interaction $g N \rightarrow g g+X$. We also consider the model presented in Ref. [1], where the cross 
section for the hadron-proton collision is viewed in a similar way as Eq. (1), where the virtual photon wave function is replaced by the corresponding wave function for the hadron projectile. The hadron-proton interaction is computed using the dipole-proton amplitude constrained by DIS data. The numerical results from both models are compared to experimental measurements focusing on the LHC kinematic regime. In the last section we discuss the main theoretical uncertainties and present the main conclusions.

\section{COLOR DIPOLE MODELS}

\section{A. Asymptotic model}

Our first investigation will consider the color dipole approach applied to the hadron-hadron collisions proposed in Ref. [3]. For simplicity, we address initially the case for proton-proton collisions in colliders. The formalism is able to provide us with the production cross section of (heavy or light) quark pairs or gluons at the final state. Namely, similarly to photon-hadron interactions, the total quark production cross section is given by $[40,41]$

$$
\begin{aligned}
\sigma(p p \rightarrow q \bar{q} X)= & 2 \int_{0}^{-\ln \left(\frac{2 m_{q}}{\sqrt{s}}\right)} d y x_{1} G\left(x_{1}, \mu_{F}^{2}\right) \\
& \times \sigma(G N \rightarrow q \bar{q} X),
\end{aligned}
$$

where $y=\frac{1}{2} \ln \left(x_{1} / x_{2}\right)$ is the rapidity of the pair and $\mu_{F} \sim$ $m_{Q}$ is the factorization scale. The quantity $x_{1} G\left(x_{1}, \mu_{F}^{2}\right)$ is the projectile gluon density at scale $\mu_{F}$ and the partonic cross section $\sigma(G N \rightarrow q \bar{q} X)$ is given by [40]

$\sigma(G N \rightarrow q \bar{q} X)=\int d z d^{2} \boldsymbol{r}\left|\Psi_{G \rightarrow q \bar{q}}(z, \boldsymbol{r})\right|^{2} \sigma_{q \bar{q} G}(z, \boldsymbol{r})$,

with $\Psi_{G \rightarrow q \bar{q}}$ being the pQCD calculated distribution amplitude, which describes the dependence of the $|q \bar{q}\rangle$ Fock component on transverse separation and fractional momentum. It is given by

$$
\begin{aligned}
\left|\Psi_{G \rightarrow q \bar{q}}(z, \boldsymbol{r})\right|^{2}= & \frac{\alpha_{s}\left(\mu_{R}\right)}{(2 \pi)^{2}}\left\{m_{q}^{2} \mathrm{~K}_{0}^{2}\left(m_{q} r\right)\right. \\
& \left.+\left[z^{2}+(1-z)^{2}\right] m_{q}^{2} \mathrm{~K}_{1}^{2}\left(m_{q} r\right)\right\},
\end{aligned}
$$

where $\alpha_{s}\left(\mu_{R}\right)$ is the strong coupling constant, which is probed at a renormalization scale $\mu_{R} \sim m_{Q}$. We notice that the wave function will lead to a dominance of dipole sizes around $r \sim 1 / m_{q}$ in the corresponding $r$ integration. Therefore, for heavy quark production, the color transparency behavior from the dipole cross section, $\sigma_{\text {dip }}(r) \propto r^{2}$, will be the main contribution (pQCD). In the charm case, an important contribution should come from the saturation region as the typical dipole size, $r \simeq 1 \mathrm{GeV}^{-1}$, can reach an order of magnitude similar to the saturation radius, $R_{s}(x)=1 / Q_{s}(x) \propto(\sqrt{s})^{-\lambda / 2}$ (with $\lambda \simeq 0.3$ ). On the other hand, for light quarks, $m_{q} \simeq 0.14 \mathrm{GeV}$, we are deep in the parton saturation (very low $x_{2}$ and small scale of probe) and nonperturbative regions. This will be the case in the following calculation.

In the partonic cross section, $\sigma_{q \bar{q} G}$ is the cross section for scattering a color neutral quark-antiquark-gluon system on the target and is directly related with the dipole cross section as follows:

$\sigma_{q \bar{q} G}=\frac{9}{8}\left[\sigma_{\text {dip }}\left(x_{2}, z \boldsymbol{r}\right)+\sigma_{\text {dip }}\left(x_{2}, \bar{z} \boldsymbol{r}\right)\right]-\frac{1}{8} \sigma_{\text {dip }}\left(x_{2}, \boldsymbol{r}\right)$.

The equation above was first derived in Ref. [15] and the main idea is that at high energies a gluon $G$ from the hadron projectile can develop a fluctuation which contains a $Q \bar{Q}$ pair. Interaction with the color field of the target then may release these heavy quarks. Such an approach is valid for high energies, where the coherence length $l_{c} \approx 1 / x_{2}$ is larger than the target radius. Hence, it is a natural framework to include the parton saturation effects and to make use of the fact that the dipole cross section is universal; i.e., it is processed independently. For the sake of completeness, the parton momentum fractions are written in terms of quark pair rapidity and masses, $x_{1,2}=$ $\frac{2 m_{Q}}{\sqrt{s}} \exp ( \pm y)$.

Following Ref. [3], we obtain the asymptotic hadronhadron total cross section within the color dipole approach considering the dominant process, $p p \rightarrow G G X$, at high energies. Now, the gluon $G$ from the projectile hadron develops a fluctuation which contains a two-gluon $(G G)$ pair which further interacts with the target's color field. Accordingly, the expression for the total cross section for gluon production at final state is given by [42]

$$
\sigma_{p p \rightarrow G G X}=2 \int_{0}^{\tilde{y}} d y x_{1} G\left(x_{1}, \mu_{F}^{2}\right) \sigma(G N \rightarrow G G X),
$$

where $\tilde{y}=-\ln \left(\frac{2 m_{G}}{\sqrt{s}}\right)$ and the effective gluon mass, $m_{G}$, has been introduced in order to regularize the calculation. Thus, in this case one has $x_{1,2}=\frac{2 m_{G}}{\sqrt{s}} \exp ( \pm y)$.

The new partonic cross section $\sigma_{G N \rightarrow G G X}$ is given by

$$
\sigma_{G N \rightarrow G G X}=\int d z d^{2} \boldsymbol{r}\left|\Psi_{G \rightarrow G G}(z, \boldsymbol{r})\right|^{2} \sigma_{G G G}(z, \boldsymbol{r}),
$$

with $\Psi_{G \rightarrow G G}$ being the corresponding distribution amplitude associated to the $|G G\rangle$ Fock state. It is obtained from Eq. (6) in the following way: $\left|\Psi_{G \rightarrow G G}\right|^{2}=$ $2\left(N_{c}-1\right)\left|\Psi_{G \rightarrow q \bar{q}}\right|^{2}$. The partonic cross section, $\sigma_{G G G}$, is the cross section for scattering a color neutral three gluon system on the target and is directly related with the dipole cross section in the following way [42]: 
$\sigma_{G G G}=\frac{1}{2}\left[\sigma_{\mathrm{dip}}\left(x_{2}, z \boldsymbol{r}\right)+\sigma_{\mathrm{dip}}\left(x_{2}, \bar{z} \boldsymbol{r}\right)+\sigma_{\mathrm{dip}}\left(x_{2}, \boldsymbol{r}\right)\right]$.

The approach described above is derived from the nonlinear $k_{\perp}$-factorization approach for the production of hard gluon-gluon dijets in gluon-hadron collisions when the coherence condition holds. This gluon-gluon dijet cross section can be investigated in different color representations and their classification in universality classes can be defined.

Now, we will present the corresponding phenomenology using Eq. (8). From Ref. [3], basically we identify two main shortcomings: the very low value for the effective gluon mass, $m_{G}=154 \mathrm{MeV}<\Lambda_{\mathrm{QCD}}$, and the identification of the scale $\mu$ with the starting evolution scale in the gluon PDFs considered, $\mu^{2}=Q_{0}^{2}$. Here, we will use the value $m_{G}=400 \mathrm{MeV}$ that is consistent with the usual values in Refs. [43-45]. Moreover, the gluon parton distribution function (PDF) probed in the low scale $\mu^{2}=m_{G}^{2}=$ $0.16 \mathrm{GeV}^{2}$ will be given by a prediction from the parton saturation physics,

$$
x G\left(x, Q^{2}\right)=\frac{3 \sigma_{0} Q_{s}^{2}}{4 \pi^{2} \alpha_{s}}\left[1-\left(1+\frac{Q^{2}}{Q_{s}^{2}}\right) e^{-\frac{Q^{2}}{Q_{s}^{2}}}\right],
$$

where updated values for the GBW model parameters have been used [28]. Consistently, for the dipole cross section we have used the GBW parametrization. It should be stressed that the result is parameter free and corresponds to the soft Pomeron contribution to the cross section.

Let us discuss quantitatively the main ingredients in the asymptotic model. For example, take the LHC energy of $\sqrt{s}=13 \mathrm{TeV}\left[\tilde{y}=-\ln \left(2 m_{G} / \sqrt{s}\right)=9.7\right.$ and $\left.\Delta y=2 \tilde{y} \simeq 19\right]$. At central gluon rapidity, $y=0$, the longitudinal momentum fractions will be $x_{1}=x_{2}=2 m_{G} / \sqrt{s} \approx 6 \times 10^{-5}$, whereas at very forward rapidity $x_{2} \simeq 4 \times 10^{-9}$. The corresponding saturation scale squared, $Q_{s}^{2}\left(x_{2}\right)$, will be $\sim 0.9 \mathrm{GeV}^{2}$ (at $y=0$ ) and $\sim 10 \mathrm{GeV}^{2}$ (at $y=\tilde{y}$ ). As $\mu^{2} \lesssim Q_{s}^{2}$, then $x G(y=0) \sim 3 \sigma_{0} Q_{s}^{2} / 4 \pi^{2} \alpha_{s} \sim 5.4 / \alpha_{s}, \sigma_{G G G} \sim$ $3 \sigma_{0} / 2$ (limit is value deep in the soft region) and $\left|\Psi_{G G}\right|^{2} \propto 2 \alpha_{s}\left(N_{c}-1\right) /(8 \pi) \delta(z-1 / 2) \delta\left(r^{2}-1 / 2 m_{G}^{2}\right)$.

This will give roughly,

$$
\frac{d \sigma_{\mathrm{tot}}}{d y}(y=0) \sim \frac{3 \sigma_{0} Q_{s}^{2}(y=0)}{16 \pi^{3}} \sigma_{0} \approx 12 \mathrm{mb},
$$

where, in the simplified expression above, the integration on rapidity is of order $2 \times 1 / \lambda \simeq 8.1$ [the rapidity dependence comes mostly from $\left.Q_{s}^{2}\left(x_{1}=2 m_{G} e^{y} / \sqrt{s}\right) \propto e^{-\lambda y}\right]$. This would give quantitatively $\sigma_{\text {tot }} \sim 95 \mathrm{mb}$, which is an order of magnitude similar to the measured cross section.

Finally, we have also considered another color dipole approach addressing the soft scattering processes. In such a case, other observables can be described as the elastic cross section and the elastic slope parameter.

\section{B. b-CGC and Eikonal models}

We follow Ref. [1] and compute the total cross section in the following way:

$$
\sigma_{\text {tot }}^{h p}(s)=2 \int d^{2} b d^{2} r d z\left|\psi_{h}(r, z)\right|^{2} N(s, r, b),
$$

which depends on the color dipole amplitude, $N(s, r, b)$, and on the hadron wave function, $\Psi_{h}(r, z)$. The expression resembles the same equation for the DIS description within the color dipole approach. That is, the photon wave function is replaced by the hadron one. Furthermore, we consider the exponential approximation of the elastic differential cross section at the diffraction peak,

$$
\left.\frac{d \sigma_{e l}}{d t} \simeq \frac{d \sigma_{e l}}{d t}\right|_{t=0} e^{B_{e l} t}=\frac{\sigma_{\mathrm{tot}}^{2}\left(1+\rho^{2}\right)}{16 \pi} e^{B_{e l} t}
$$

where $t=-q_{t}^{2}$ is the momentum transfer in a $h p$ collision, $\rho$ is the real-to-imaginary ratio of the forward elastic amplitude

$$
\rho^{h p}(s)=\frac{\operatorname{Re} A_{e l}(s, t=0)}{\operatorname{Im} A_{e l}(s, t=0)} \simeq \frac{\pi}{2 \sigma_{\mathrm{tot}}^{h p}} \frac{d \sigma_{\mathrm{tot}}^{h p}}{d \ln \left(s / s_{0}\right)},
$$

and the slope, $B_{e l}^{h p}(s)=B_{0}+B^{\prime}(s)$ with $B_{0}=7.8 \mathrm{GeV}^{-2}$ and

$$
B^{\prime}(s)=\frac{\int b^{2} d^{2} b d^{2} r\left|\psi_{h}(r)\right|^{2} N(r, b, x)}{\sigma_{\text {tot }}}=\frac{1}{2}\left\langle b^{2}\right\rangle .
$$

In Eq. (15) we invoke a first order derivative dispersion relation to provide an estimate of the parameter $\rho$ at LHC energies, especially at $13 \mathrm{TeV}$. Once the leading terms in the amplitude of dipole models are interpreted here in the Regge language as Pomeron terms (soft + hard), we will have not accounted for Odderon signatures. Therefore, our predictions for $p p$ and $\bar{p} p$ observables are degenerate (the same being true for $\pi^{ \pm} p$ ).

Finally, the elastic cross section can be computed by integrating Eq. (14) to give (as $\rho^{2} \ll 1$ )

$$
\sigma_{e l}^{h p}(s) \simeq \frac{\left[\sigma_{\mathrm{tot}}^{h p}(s)\right]^{2}}{16 \pi B_{e l}^{h p}(s)} .
$$

Here, in the meson-proton scattering the meson is treated as a $q \bar{q}$ pair and the calculations follow that of DIS; i.e., the interaction of a color dipole with a proton target and saturation physics can be embedded in the dipole amplitude. Similar approaches have also been considered in Refs. [46,47], where the Pomeron dynamics is written in terms of the dipole-dipole cross section. For instance, in Ref. [46] the large dipoles are dominated by a soft Pomeron contribution, whereas small dipoles are driven by a hard 
Pomeron. On the other hand, in Ref. [47], based on Mueller's cascade model, the authors discuss several contributions including the effect of Pomeron loops.

For the wave functions of mesons and baryons, we use the phenomenological ansatz by Wirbel-Stech-Bauer [46], which gives

$$
\psi_{h}(z, r)=\sqrt{\frac{z(1-z)}{2 \pi S_{h}^{2} N_{h}}} \exp \left(-\frac{\left(z-\frac{1}{2}\right)^{2}}{4 \Delta z_{h}^{2}}-\frac{r^{2}}{4 S_{h}^{2}}\right),
$$

where the hadron wave function is normalized to unity

$$
\int d z d^{2} r\left|\psi_{h}(z, r)\right|^{2}=1
$$

This condition yields the following normalization constant, $N_{h}$ :

$$
N_{h}=\int_{0}^{1} d z z(1-z) e^{-\left(z-\frac{1}{2}\right)^{2} / 2 \Delta z_{h}^{2}}
$$

Therefore, mesons and baryons are assumed to have a $q \bar{q}$ and quark-diquark valence structure. As quark-diquark systems are equivalent to $q \bar{q}$ systems, this allows us to model not only mesons but also baryons as color dipoles. The values of parameters in our case are the following: $\Delta z_{h}=0.3(0.2)$ and $S_{h}=0.86(0.607) \mathrm{fm}$, for $p / \bar{p}\left(\pi^{ \pm}\right)$, respectively [46]. $S_{h}$ is a fit parameter which gives a measure of the transverse hadronic radius. Hence, as the hadron wave function has a Gaussian profile, which is centered at $S_{h}$ [see Eq. (18)], it is expected that dipoles with approximately the hadron radius dominate the contribution to the cross sections.

At this point, some discussion is in order. In our calculations the proton is considered as a quark-diquark system having a mesonlike structure. Specifically, the proton can be viewed as a bound state of an up quark and an isospin zero, quark spin zero spatially extended twoquark state, the diquark. In this case, quark-diquark systems are equivalent to quark-antiquark systems and we accordingly obtain $\langle z\rangle=1 / 2$ from the wave function for protons in Eq. (18). The three-quark structure of a baryon makes the model notably complex but produces similar phenomenological results as in the quark-diquark picture. A comparison between the three-body picture and the diquark one for protons concerning soft observables has been done in Ref. [48] (see, e.g., Fig. 1 and Table III in [48]). On the other hand, in the literature different treatments for the baryon wave function are considered. For instance, in Ref. [49] the proton wave function is obtained by the symmetric oscillator wave function of the valence threequark proton. In this approximation, the proton is viewed as a $3 / 2$ color dipole spanned between quark pairs. The distribution of the size of color dipoles with transverse size $r$ spanned between $q \bar{q}$ in the proton is considered Gaussian,

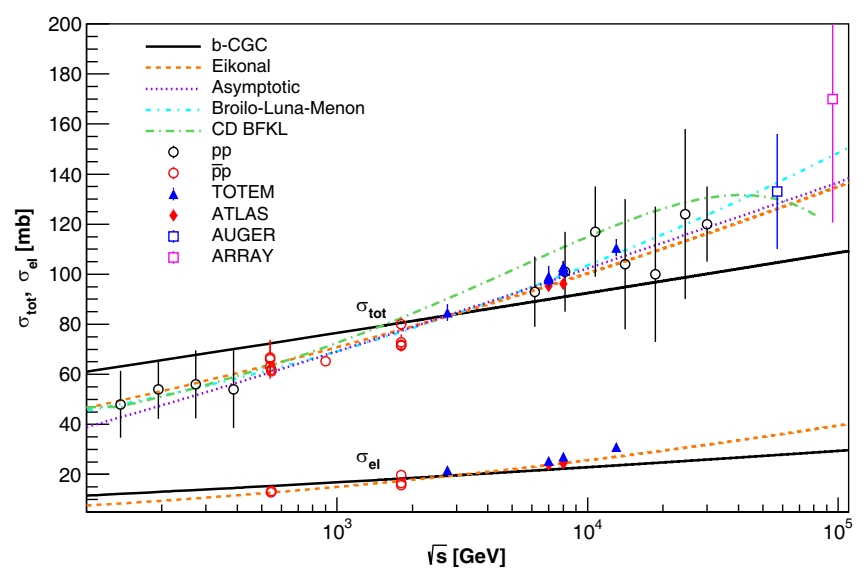

FIG. 1. The total and elastic cross sections for $p p$ collisions. The upper cross sections are total cross sections, while the lower ones are the elastic cross sections. Tevatron, SPS, LHC, and cosmic rays data are presented [30-37,50,51]. The lines are results from models considered with the fitted parameter $Q_{0}^{2}$.

where $\left\langle r_{p}^{2}\right\rangle=0.658 \mathrm{fm}^{2}$. This value is not far from the value $S_{h}^{2}=0.740 \mathrm{fm}^{2}$ appearing in Eq. (18) for protons. Accordingly, the average $\langle z\rangle=1 / 3$ is obtained. However, as we will see afterwards, the calculation of a low mass (LM) single diffraction cross section becomes a hard task as three color centers from constituent quarks should be used and the average amplitude squared, $\left\langle N^{2}\right\rangle$, has to be taken in each of these configurations.

Before discussing an impact-parameter dipole amplitude extracted from DIS data, we would need to rewrite the energy dependence from photon-hadron scattering in terms of the appropriate Bjorken scaling variable $x$. In this work, the following ansatz has been considered:

$$
\frac{1}{x}=\frac{s r^{2}}{\left(s_{0} R_{c}^{2}\right)}
$$

which has been successfully considered in Ref. [52] and where $s_{0}^{2} \sim m_{h}^{2}$ and $R_{c}=0.2 \mathrm{fm}$. Such an ansatz is numerically equivalent to the proposal $\frac{1}{x}=\frac{s}{Q_{0}^{2}}$, with $Q_{0}^{2} \sim\left(2 m_{q}\right)^{2} \simeq m_{h}^{2}$, made in Ref. [1]. For simplicity and faster numerical calculation, we consider the last relation, where $Q_{0}^{2}$ is a free parameter to be fitted to the total crosssection data, above $\mathrm{cm}$ energies $\sqrt{s} \gtrsim 100 \mathrm{GeV}$.

For the impact-parameter amplitude, we first consider the parametrization based on the color glass condensate ideas (called from now on the b-CGC model). In the b-CGC model, the color dipole-proton amplitude is given by

$N(x, r, b)=\left\{\begin{array}{ll}N_{0}\left(\frac{r Q_{s}}{2}\right)^{2 \gamma_{\text {eff }},} & r Q_{s} \leq 2 \\ 1-\exp \left[-\mathcal{A} \ln ^{2}\left(\mathcal{B} r Q_{s}\right)\right], & r Q_{s}>2\end{array}\right.$,

where the effective anomalous dimension and the saturation scale, $Q_{s}$, are defined as 


$$
\begin{gathered}
\gamma_{\mathrm{eff}}=\gamma_{s}+\frac{1}{\kappa \lambda Y} \ln \left(\frac{2}{r Q_{s}}\right), \\
Q_{s}=\left(\frac{x_{0}}{x}\right)^{\frac{\lambda}{2}} \exp \left\{-\frac{b^{2}}{4 \gamma_{s} B_{C G C}}\right\},
\end{gathered}
$$

where, accordingly, $\quad Y=\ln (1 / x)$ and $\kappa=\chi^{\prime \prime}\left(\gamma_{s}\right) /$ $\chi^{\prime}\left(\gamma_{s}\right)=9.9$, with $\chi$ being the leading-order BFKL characteristic function. The updated values for the model's parameters are the following: $B_{C G C}=5.5 \mathrm{GeV}^{-2}, \gamma_{s}=$ $0.6492, N_{0}=0.3658, x_{0}=6.9 \times 10^{-4}$, and $\lambda=0.2023$, which have been published in Ref. [53]. That fit was performed in the range $x \leq 0.01$ and $Q^{2} \in[0.75,650] \mathrm{GeV}^{2}$, with $m_{c}=1.4 \mathrm{GeV}$, using high precision combined HERA data.

We have also tried an eikonal-like expression for the dipole amplitude, which has a different impact-parameter dependence. The function $S(b)$ is now described by the dipole-profile function. Namely, the amplitude has the following form:

$$
N(x, r, b)=1-\exp \left(-\frac{1}{2} \hat{\sigma}(x, r) S(b)\right)
$$

with

$$
\begin{gathered}
\hat{\sigma}(x, r)=\sigma_{0} \frac{\left(r Q_{s}(x)\right)^{2}}{4}, \\
S(b)=\frac{2 \beta b}{\pi R^{2}} K_{1}(\beta b) .
\end{gathered}
$$

Moreover, we have considered the parameters for $\hat{\sigma}$ from the GBW saturation model [28], taking $R^{2}=4.5 \mathrm{GeV}^{-2}$ and $\beta=\frac{\sqrt{8}}{R}$.

The eikonal-like model above is strongly inspired in the success obtained in Ref. [54], where a universal expression of cross sections for the exclusive vector meson production and DVCS in photon-proton and photon-nucleus interactions based on the geometric scaling phenomenon has been obtained. Using the same form, Eq. (24), a theoretical parametrization based on the scaling property where cross sections depend only on the single variable $\tau=\left(\mu^{2} / Q_{s}^{2}\right)\left(\mu^{2}=Q^{2}+m_{V}^{2}\right.$ for vector mesons and $\mu^{2}=Q^{2}$ for DVCS, respectively) was found. In that work, the saturation scale controls the energy dependence and nuclear effects as well. The eikonal-like model then describes all available data from DESY-HERA for $\rho, \phi, J / \psi$ production and DVCS measurements. Furthermore, the photonuclear cross sections for $\rho$ and $J / \psi$ production extracted from the ultraperipheral heavy ion collisions at the LHC; i.e., $\sigma(\gamma P b \rightarrow \mathrm{VM}+P b)$, are also quite well described.
TABLE I. Best fit parameter $Q_{0}^{2}$ and $\chi^{2} /$ d.o.f. of fits to $p p$ and $\pi^{+} p$ total cross section data for b-CGC and Eikonal models.

\begin{tabular}{lcc}
\hline \hline Model & $Q_{0}^{2}\left[\mathrm{GeV}^{2}\right]$ & $\chi^{2} /$ d.o.f. \\
\hline b-CGC $(p p)$ & $(9.44 \pm 0.57) \times 10^{-5}$ & $518.40 / 22=23.6$ \\
b-CGC $\left(\pi^{+} p\right)$ & $0.10 \pm 0.12$ & $9.88 / 6=1.65$ \\
Eikonal $(p p)$ & $0.308 \pm 0.019$ & $70.25 / 22=3.19$ \\
Eikonal $\left(\pi^{+} p\right)$ & $13 \pm 14$ & $9.25 / 6=1.54$ \\
\hline \hline
\end{tabular}

\section{FIT RESULTS AND DISCUSSION}

\section{A. Total and elastic cross sections}

Fits to the $p p$ and $\bar{p} p$ total cross sections for the three models presented in last section are shown in Fig. 1. Both accelerator and cosmic ray data have been gathered from the PDG2018 review [29], recent LHC measurements, mostly by TOTEM and ATLAS Collaborations [30-37] as well as from the Auger and Telescope Array Collaborations [50,51]. All fits have been performed using the TMINUIT class of the ROOT framework [55] through the MIGRAD algorithm. Specifically, we minimize the total cross section data, $p p, \bar{p} p$, and $\pi^{+} p$ scatterings, for $\sqrt{s} \geq 100 \mathrm{GeV}$, using the chi squared per degrees of freedom (d.o.f.), $\chi^{2} /$ d.o.f., criterium as a goodness of fit estimator. As previously mentioned, the asymptotic model has only fixed parameters and for b-CGC and the Eikonal models, the only fit parameter to be tuned is $Q_{0}^{2}$. Best fit parameters of these models are thus given in Table I.

As shown in Figs. 1 and 2, the asymptotic model provides a reasonable description of the data in the wide energy range, $100 \mathrm{GeV}<\sqrt{s}<13 \mathrm{TeV}$. This feature can be related to the dominant role of gluon production at very low $x$, as the model has only four fixed parameters, namely, $m_{G}, \sigma_{0}, x_{0}$, and $\lambda$.

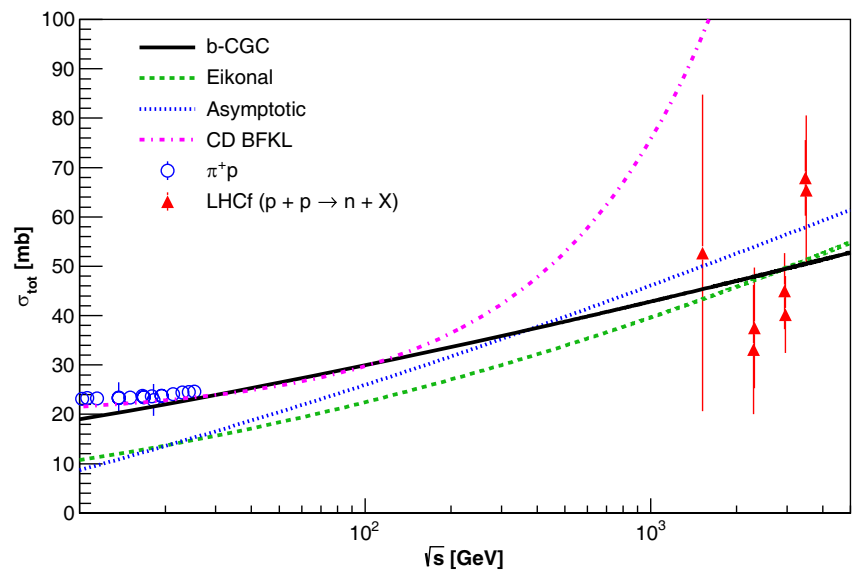

FIG. 2. The total cross section for $\pi^{+} p$ collisions. Data are obtained from inclusive leading neutron spectra in the LHCf Collaboration of the LHC [56,57]. Low energy data are also presented. 
On the other hand, the b-CGC model gives a slowly rising total cross section, with a preasymptotic form $\sigma_{\text {tot }} \sim a \ln s$. Such behavior is related to the fact that dipoles with sizes nearly the proton radius dominate once the hadron wave function has a Gaussian profile centered at $R_{p}$. For $r Q_{s} \geq 2$, the dipole cross section in b-CGC is mainly driven by the Balitsky-Kovchegov (BK) asymptotic solution [see Eq. (21)]. At high energies (small $x$ ) the saturation scale grows. Thereby, the quantity $r Q_{s}$ becomes larger and the dipole amplitude tends to the unity, which leads to the saturation regime. At LHC and cosmic rays energies, the system is saturated and the cross section has already reached its limit.

Some words of caution are in order at this point. Most saturation models [b-CGC, impact-parameter saturation model (IPSAT), Golec-Biernat Wusthoff (GBW), and so on] predict a total cross section proportional to quantity $\sigma_{0} \sim 27 \mathrm{mb}$ which is quite small compared to the typical values of the measured cross section (even for the pion case). This was discussed already in Ref. [1] for the GBW model and the numerical solution of the BK equation. The situation is similar here for the b-CGC model, where the smallness of overall normalization had to be compensated by an unrealistic value of the $Q_{0}^{2}$ parameter in $p p$ case (see Table I). The situation is different for the Eikonal model, where the overall normalization is given by the integration over impact parameter of the profile $S(b) \propto b K_{1}(\beta b)$, which corresponds to the proton dipole form factor in the momentum transfer representation.

Regarding the Eikonal model, the profile function considered, $S(b) \sim \frac{b}{R} K_{1}\left(\frac{b}{R}\right)$, results in an asymptotic total cross section, $\sigma_{\text {tot }} \sim \ln ^{2} s$, as long as $1 / x \sim s$, as stated by the ansatz (20) [1]. This Froissart-bounded cross section can be naturally obtained in structure function models with the leading asymptotic form $F_{2}(x) \sim \ln ^{2}(1 / x)$, at low $x$, such as in the model by Block-Durand-Ha [58,59], whose analytical form ultimately leads to the dipole cross section given in Ref. [3]. Thus, a deep link between the dipole cross section (an its sizes) and the total cross section cannot only be anticipated at very high energies, but it is essential to understand low- $x$ parton dynamics. In addition, the same asymptotic energy behavior is seen in soft Pomeron models, such as BLM [60], COMPETE [61], and PDG [29].

To establish a direct comparison with other popular models of current Regge phenomenology we also plot in Fig. 1 the prediction of the model by Broilo-Luna-Menon (BLM) [60], in which the energy dependence of the soft Pomeron is parametrized as follows (model II):

$$
\sigma_{\mathrm{tot}}^{\mathbb{P}}(s)=A+D \ln ^{2}\left(s / s_{0}\right),
$$

where $A=29.6 \pm 1.2 \mathrm{mb}, D=0.251 \pm 0.010 \mathrm{mb}$, and $s_{0}=4 m_{p}^{2} \simeq 3.521 \mathrm{GeV}^{2}$. As this model is inspired in the COMPETE analysis (pre-LHC) [61-63] we shall refer to it as the BLM model.

We have also estimated the pion-proton total cross section. Our predictions are shown in Fig. 2 compared to recently extracted data from leading neutron production in the $\mathrm{TeV}$ region $[56,57]$ by using recent data from the LHCf Collaboration [64]. The magnitude and energy evolution predicted by the models tested is in quite good agreement with the data, despite their large error bars.

For the asymptotic model, we use the additive quark model, where $\sigma_{\text {tot }}^{\pi N} / \sigma_{\text {tot }}^{N N}=2 / 3$. Regarding the b-CGC and Eikonal models, we explicitly take into account $\left|\psi_{\pi}(z, r)\right|^{2}$ from Eq. (18). The low energy data are also presented. In the models discussed so far only the Pomeron contribution is being computed. For low energy a nonperturbative contribution as well as the Reggeon piece have to be added.

Predictions of models for the total cross section at LHC energies of $7,8,13$, and $14 \mathrm{TeV}$ and at the cosmic ray energies, $57 \mathrm{TeV}$ (Pierre Auger Observatory) and $95 \mathrm{TeV}$ (Telescope Array), are shown in Table II. It is important to mention that we have not presented the b-CGC predictions because they did not have a good agreement with data, as it can be clearly seen in Fig 1. Thereby, for the observables calculated in the next sections, we will not take into account the results presented by this model.

Before analyzing the $\rho$ parameter and hadronic forward slope in next subsection, we explicitly compare our predictions to the color dipole BFKL-Regge expansion (CDBFKL) approach by Fiore et al. (Ref. [39] and references therein). The main ingredient in this formalism is the BFKL dipole cross section, $\sigma\left(Y=\ln \left(x_{0} / x\right), r\right)$, which sums the $\sim \alpha_{s} \ln (1 / x)$ multigluon production cross sections in perturbative QCD. The initial condition for the evolution at $x=x_{0}$ and dipoles having transverse size $r$ is the Yukawa screened two-gluon exchange. The evolution equation for the dipole cross section concerning the nonunitarized running $\mathrm{CD}$ BFKL amplitudes is given by,

$$
\begin{aligned}
\frac{\partial \sigma(Y, r)}{\partial Y}= & \int d^{2} \vec{r}_{1}\left|\psi\left(\vec{r}_{1}\right)-\psi\left(\vec{r}_{2}\right)\right|^{2} \\
& \times\left[\sigma_{3}\left(Y, \vec{r}, \vec{r}_{1}, \vec{r}_{2}\right)-\sigma(Y, r)\right]
\end{aligned}
$$

where $\psi(\vec{r}) \propto \frac{\hat{r}}{R_{c}} K_{1}\left(r / R_{c}\right)$ is the radial light-cone wave function of the dipole with the vacuum screening of

TABLE II. Predictions of $\sigma_{\mathrm{tot}}^{p p / \bar{p} p}$ for the asymptotic and eikonal models.

\begin{tabular}{lcc}
\hline \hline$\sqrt{s}(\mathrm{TeV})$ & Asymptotic: $\sigma_{\mathrm{tot}}^{p p / \bar{p} p}(\mathrm{mb})$ & Eikonal: $\sigma_{\mathrm{tot}}^{p p / \bar{p} p}(\mathrm{mb})$ \\
\hline 7.0 & 96.9 & 95.4 \\
8.0 & 99.0 & 97.2 \\
13 & 106 & 104 \\
14 & 107 & 105 \\
57 & 128 & 126 \\
95 & 136 & 134 \\
\hline \hline
\end{tabular}


infrared gluons (infrared cutoff regulator is $R_{c}=0.26 \mathrm{fm}$ ). The $q \bar{q} g$-nucleon three-parton cross section, Eq. (7), is a function of $\vec{r}_{1,2}$, which are, respectively, the quark-gluon and antiquark-gluon transverse separations in the twodimensional impact-parameter plane for dipoles generated by the quark-antiquark color dipole source.

In [39], the unitarity absorption corrections are computed using the BK nonlinear BFKL equation in the impactparameter representation. The evolution equation in this case reads as

$$
\begin{aligned}
\frac{\partial \sigma(Y, r)}{\partial Y}= & \int d^{2} \vec{r}_{1}\left|\psi\left(\vec{r}_{2}\right)-\psi\left(\vec{r}_{1}\right)\right|^{2} \\
& \times\left\{\sigma\left(Y, \vec{r}_{1}\right)+\sigma\left(Y, \vec{r}_{2}\right)-\sigma(Y, r)\right. \\
& \left.-\frac{\sigma\left(Y, \vec{r}_{1}\right) \sigma\left(Y, \vec{r}_{2}\right)}{4 \pi B_{12}} \exp \left[-\frac{r^{2}}{8 B_{12}}\right]\right\},
\end{aligned}
$$

where $B_{12}=B_{1}\left(Y, r_{1}\right)+B_{2}\left(Y, r_{2}\right)$ and $B_{i}=B\left(Y, r_{i}\right)$. The authors consider that the diffraction cone slope $B$ drives the area populated with interacting gluons. Specifically, the diffraction slope for the forward cone in the dipole-nucleon scattering is given by the expression $B(Y, r)=\left(r^{2} / 8\right)+\left(R_{N}^{2} / 3\right)+2 \alpha_{I P}^{\prime} Y$, with $R_{N}^{2} \simeq 12 \mathrm{GeV}^{-2}$ and $\alpha_{I P}^{\prime} \approx 0.1 \mathrm{GeV}^{-2}$.

Accordingly, in Fig. 1 the predictions of Fiore et al. [65] for the total cross section (green dot-dashed curve) are shown. We see that absorptive corrections are strong at cosmic rays interaction and at the highest collision of the LHC. Up to $2 \mathrm{TeV}$ their results is very similar to ours. For completeness, we also add the predictions from the CD BFKL approach (without absorption corrections) for the pion-proton cross section in Fig. 2. The dot-dashed curve gives the hard contribution to $\pi N$ cross section taken from Ref. [49] [Eq. (28) and parameters in Table I from that reference]. The low energy data is nicely described and the high energy LHC values could be reproduced in case absorption is included. The absorption effect should be similar to the proton case in the same energy range.

\section{B. Real-to-imaginary ratio and the forward slope}

Furthermore, we also give predictions for two forward energy-dependent observables: (i) $\rho^{p p, \bar{p} p}(s)$, the real-toimaginary ratio of the elastic amplitude, which follows in Fig. 3, and (ii) $B_{e l}^{p p, \overline{p p}}(s, t=0)$ the forward slope, which is shown in Fig. 4. Both plots comprise very recent LHC data and especially for $\rho$, an adequate description of the LHC13 datum (within error bars) is achieved. On the one hand, predictions from dipole models deviate significantly from the data, especially the b-CGC model. Such behavior is related to the very rapid decrease of the $b$ distribution at large impact parameters, $N(r, x, b) \sim 1-$ $\exp ^{-\alpha(r, x) b^{4}}$, which approximately follows a black-disk

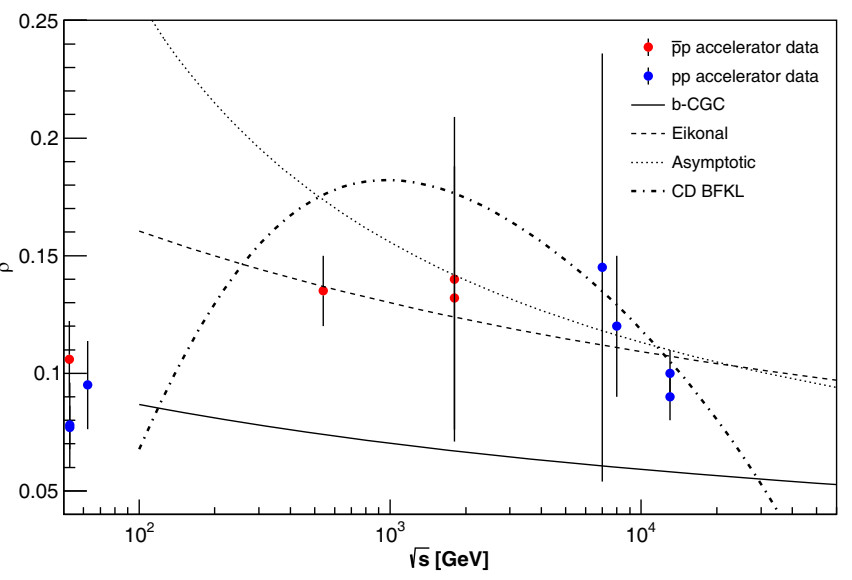

FIG. 3. Real-to-imaginary ratio predictions the b-CGC, Eikonal, and asymptotic models and prediction from the CD BFKL approach together with recent LHC data [32].

shape, $N(r, x, b) \sim \Theta(b-R)$, and leads to an almost flat energy dependence of $B_{e l}$. We recall that such behavior is very similar to those presented in Ref. [1], where the GBW and Glauber-Mueller models for the dipole cross section were considered. In Ref. [65] no prediction is done for the forward slope; however, an estimation can be done using the forward cone in the dipole-nucleon scattering. Taking for simplicity that the average dipole size is $\langle r\rangle \approx \sqrt{\left\langle r_{p}^{2}\right\rangle}$, the corresponding slope is $B_{\text {dip }}(s,\langle r\rangle) \approx$ $\left(\left\langle r_{p}^{2}\right\rangle / 8\right)+\left(R_{N}^{2} / 3\right)+2 \alpha_{I P}^{\prime} \ln \left(s x_{0} / m_{\rho}^{2}\right)$. At $7 \mathrm{TeV}$ a rough estimation is $B_{e l}=B_{0}+B_{\text {dip }} \approx(7.8+8.87) \mathrm{GeV}^{-2} \simeq$ $17 \mathrm{GeV}^{-2}$ which is close to the TOTEM measurement $\left(B_{e l}^{\exp }(7 \mathrm{TeV})=19.9 \pm 0.3 \mathrm{GeV}^{-2}\right)$.

Conversely, due to the smoother $b$ distribution given in Eqs. (24) and (26), the Eikonal model shows a better agreement with data, yielding a more acceptable trend of rising for $B_{e l}(s)$. Indeed, as we show in Fig. 5, predictions of this model for the elastic differential cross section reproduce the general structure of the diffraction cone

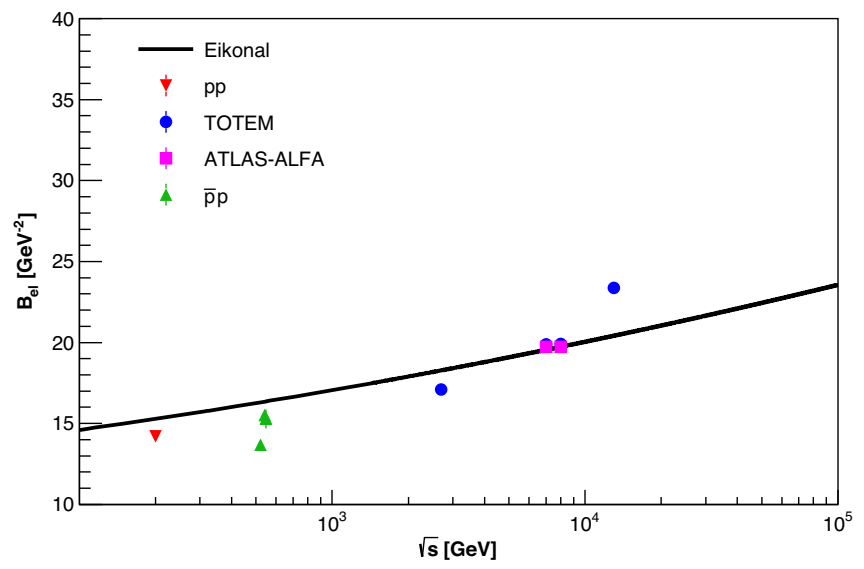

FIG. 4. Energy dependence of the slope, $B_{e l}^{p p}$, predicted by the Eikonal model. 


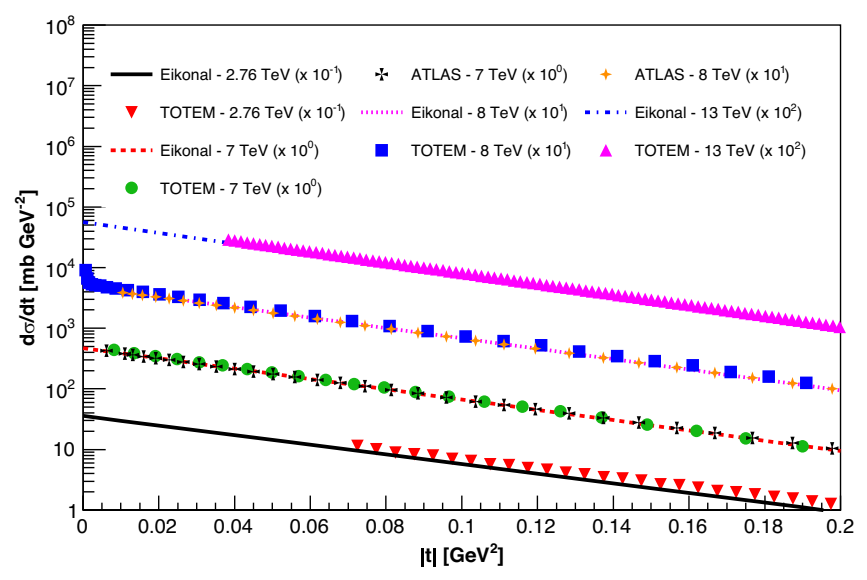

FIG. 5. Differential elastic cross-section data measured by the ATLAS and TOTEM Collaborations at $\sqrt{s}=2.76$ [66], 7, [35,67], 8 [33,34], and $13 \mathrm{TeV}$ [68] and predictions of the Eikonal model in the region $0<-t \lesssim 0.2 \mathrm{GeV}^{2}$.

$\left(0<-t \lesssim 0.2 \mathrm{GeV}^{2}\right)$ at LHC energies, especially at 7.0 and $8.0 \mathrm{TeV}$.

Finally, we present the corresponding $\rho$ parameter for the CD BFKL model based on the results for the total $p p$ cross section and make use of dispersion relations (derivative dispersion relation). It is shown in Fig. 3 (black dot-dashed curve), which is driven by the change of inflexion in the total cross section in the high energy collider region. The normalization is still in agreement with LHC data, whereas the shape shows somewhat of a disagreement.

\section{Low mass diffraction}

For incorporating color transparency in a natural way, color dipole models are a perfect framework to study inelastic diffraction. Indeed, color dipoles can be regarded as eigenstates of diffraction [69].

In the one-channel models we have developed so far, low mass inelastic diffractive eigenstates can be treated using the Good-Walker (GW) mechanism [70]. Since diffraction arises from fluctuations in high-energy scattering amplitude, we calculate the contribution of color dipoles to the single diffractive cross section in the LM region through the following relation:

$$
\sigma_{\mathrm{SD}}^{\mathrm{LM}}(s)=\left\langle N^{2}\right\rangle-\langle N\rangle^{2},
$$

where

$$
\begin{aligned}
\left\langle N^{2}\right\rangle & =\left(\int d^{2} b \int d z d^{2} r\left|\Psi_{h}(r, z)\right|^{2} N^{2}\right), \\
\langle N\rangle & =\int d^{2} b\left(\int d z d^{2} r\left|\Psi_{h}(r, z)\right|^{2} N\right) .
\end{aligned}
$$

The first term in Eq. (30) encompasses the quasielastic cross-section term, where excitations of the target (beam) particle can occur in the interaction with dipoles within the proton. The second term corresponds to the pure elastic scattering term. The predictions for the dipole model are presented in Fig. 6, as a function of the center-of-mass energy. The theoretical curve (we choose the Eikonal model as reference) is compared to non-LHC collider data (ISR [71], UA4 [72], UA5 [73], E710 [74] and CDF [75]) and the recent LHC measurements. In particular, we consider the ALICE data [76] at $\sqrt{s}=0.9,2.76$, and $7 \mathrm{TeV}$ $\left(M_{X}<200 \mathrm{GeV} / \mathrm{c}^{2}\right)$, the measurements of TOTEM [77] $\left(3.4<M_{X}<1100 \mathrm{GeV} / \mathrm{c}^{2}\right)$ and CMS [78] $\left(12<M_{X}<\right.$ $\left.394 \mathrm{GeV} / \mathrm{c}^{2}\right)$, as well. An approach similar to ours is presented in Ref. [79], where fluctuations in the BFKL ladder are taken into account. It was demonstrated that in high energy proton-proton collisions these fluctuations are strongly suppressed by parton saturation.

The Good-Walker formalism was originally conceived so as to describe a system of a nucleon plus its diffractive $N^{*}$ isobars. Clearly, this simplistic approach is not suitable for high energy diffraction where $M_{\text {diff }}^{2}$ is bounded by $0.05 s$, leading to a continua of diffractive Fock states [80].

GW models shortcomings are amended once multiPomeron interactions are included, leading to a high mass diffraction [81]. If we consider a single diffractive channel $p+p \rightarrow p+M_{\mathrm{SD}}$, Mueller's triple Pomeron mechanism yields high single diffractive (SD) mass which is non-GW [80].

$\mathrm{CDF}$ analysis suggests a relatively large value for $G_{3 I P}$. Therefore, it is necessary to consider a very large family of multi-Pomeron interactions (enhanced $I P$ ) which are not included in the GW formalism. This dynamical feature becomes significant above Tevatron energy and leads to profound differences in the calculated values of soft cross sections. As expected, it can be seen in Fig. 6 that GW formalism does not show good results at high energies due to the fact that high mass diffraction is not taken into

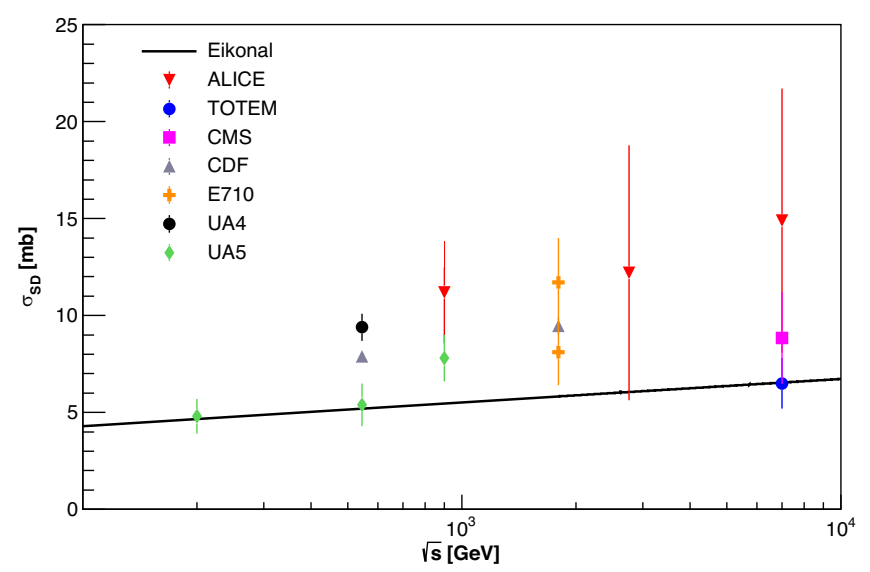

FIG. 6. SD cross section for the reaction $p p / \bar{p} \rightarrow p X$ as a function of the center-of-mass energy, $\sqrt{s}$. The curve is the result for the color dipole amplitude from Eikonal model and considers only the low mass diffraction contribution. 
account. The GW approach just considers elastic processes, i.e., on the forward direction. Hence, only the low mass diffraction is taken into account. However, at high energies the high mass contribution plays a significant role as peripheral regions of the hadrons; i.e., inelastic processes cannot be neglected in the computing of $\sigma_{\mathrm{SD}}$.

Despite the high mass, diffractive dissociation is out of the scope for the present study and it can be properly addressed in a color dipole approach. For example, previously before both low and high mass excitations were described by the Good-Walker mechanism in Ref. [79]. In that work, the high mass diffraction is connected to fluctuations in the BFKL evolution and it is shown that in $p p$ collisions unitarity constraints and saturation decrease those fluctuations towards the black-disc limit of the scattering process. Moreover, the dipole cascade model can reproduce the expected triple-Regge form for the bare Pomeron with $\alpha_{I P}(0)=1.21$ and $\alpha_{I P}^{\prime}=0.2 \mathrm{GeV}^{-2}$, and the triple-Pomeron coupling is shown to be almost constant, $g_{3 I P} \approx 0.3 \mathrm{GeV}^{-1}$. It is argued that $\mathrm{GW}$ and triplePomeron formalisms for high mass dissociative diffraction are just different aspects of the same phenomenon. Specifically, in both approaches, diffractive excitation is the shadow of absorption into inelastic channels. This conclusion is not completely new as in the seminal work in Ref. [82] where a direct computation of the triplePomeron coupling for both diffractive photoproduction and DIS at large $Q^{2}$ has been done within the CD BFKL formalism already discussed. A weak dependence on $Q^{2}$ was found, producing $G_{3 I P}\left(Q^{2}\right) \approx 0.23 \mathrm{GeV}^{-2}$ at $Q^{2}=0$ and $G_{3 I P}\left(Q^{2}\right) \approx 0.36 \mathrm{GeV}^{-2}$ for $Q^{2} \geq 3 \mathrm{GeV}^{2}$. In the context of the formulations presented here, within the color dipole picture the high mass dissociation can be understood as a three stage process. First, the penetration of the projectile dipole through the target without inelastic interaction then followed by the emission of one extra gluon (considered a new dipole in large $N_{c}$ limit). Finally, one has the interaction of two produced dipoles with the target. The main ingredient in the last stage is the amplitude of gluon-dipole scattering that has been investigated in Ref. [83]. Starting from the dipole amplitude in Eq. (13) written in terms of the opacity function $\Omega$, one has $N(s, r, b)=1-\exp \left[-\frac{1}{2} \Omega(s, r, b)\right]$. For instance, in our eikonal-type model, Eq. (24), $\Omega(s, r, b)=\hat{\sigma}(x, r) S(b)$. For the proton considered as an effective color dipole (the quark-diquark picture) the high mass diffraction cross section reads as

$$
\begin{aligned}
M^{2} \frac{\sigma_{\mathrm{SD}}^{\mathrm{HM}}(s)}{d M^{2}}= & \frac{\alpha_{s} N_{c}}{2 \pi} \int d^{2} b d z d^{2} r\left|\psi_{p}(r, z)\right|^{2} e^{-\Omega(s, r, b)} \\
& \times\left[e^{-\Omega(s, r, b)} r^{2} I_{1}\left(\frac{s}{M^{2}}, r, b\right)-r^{2} I_{2}(s, r, b)\right],
\end{aligned}
$$

with the following auxiliary integrals:

$$
\begin{aligned}
I_{1}= & \int_{r^{2}}^{\infty} \frac{d r^{\prime 2}}{r^{\prime 4}}\left\{1-\exp \left[-\left(\Omega\left(\frac{s}{M^{2}}, r^{\prime}, b\right)\right.\right.\right. \\
& \left.\left.\left.-\frac{1}{2} \Omega\left(\frac{s}{M^{2}}, r, b\right)\right)\right]\right\}^{2}, \\
I_{2}= & \int \frac{d^{2} r^{\prime}}{2 \pi r^{\prime 2}\left(\vec{r}-\vec{r}^{\prime}\right)^{2}}[1-\exp (-\Omega(s, r, b))]^{2},
\end{aligned}
$$

where $I_{2}$ is related to a change for the elastic scattering of the original dipole (with transverse size $r$ ) due to the emission of an extra gluon. In $I_{1}$ the expression in the curly bracket is the amplitude for gluon-dipole scattering [83]. Applications of the above formalism to the $p p$ scattering will be postponed for future studies.

As a final comment on the expression, Eq. (30), for the low-mass contribution to the SD cross section, we see that it is suitable for computing the corresponding proton-nucleus $(p A)$ cross section. This can be performed by replacing the proton profile function $S(b)$ in our case by the one extracted from nuclear form factors, $S_{A}(b)$ (Woods-Saxon or similar parametrizations). The investigation about the size of nuclear effects in a single diffraction is an open question in the literature. For instance, in Ref. [84] predictions for the $\mathrm{SD}$ cross section in $p P b$ collisions at the LHC are obtained in the context of the Glauber model for nuclear scatterings and take into account Regge phenomenology (including an effective Pomeron flux, which describes the measured SD cross section in $p p$ collisions). Recently, in Ref. [85] the authors investigate the diffractive excitation in $p A$ collisions based on the dynamics of relativistic nuclear collisions through the concept of hadronic cross-section fluctuations. These fluctuations are related to inelastic shadowing and diffractive dissociation and their effect decreases at larger energies and heavier nuclei.

\section{SUMMARY}

In summary, we have applied to soft hadron-hadron scattering the color dipole picture including the parton saturation phenomenon as the transition region between soft and hard domains. We have shown that the inclusive process is mainly driven for dipole sizes near the saturation radius in the high energy regime. The main advantage is that the corresponding phenomenology is almost free of parameters as they are completely constrained from DIS data in $e p$ interactions. The models rely on the dipole cross section or the $b$-dependent dipole amplitude and indicate that the impact-parameter profile is crucial for a good data description. In this context, our best results followed from the eikonal model, for which a smoother impact-parameter structure was built. In fact, the wealth of high energy elastic scattering data can be nicely described by this model, including $\sigma_{\mathrm{tot}}, \sigma_{e l}, \rho, d \sigma_{e l} / d t$ in the diffraction cone and $\sigma_{\mathrm{SD}}$ in the low mass region, using a one-channel eikonal approach. These findings indicate a possible path of 
exploring even further the color dipole formalism as an alternative approach to the more traditional ReggePomeron calculus to handle soft hadron-hadron and hadron-nucleus scattering processes, where, for instance, the role of multiple parton interactions can be properly addressed. We are currently investigating this possibility.

\section{ACKNOWLEDGMENTS}

This work was partially financed by the Brazilian funding agencies CNPq and CAPES. D. A.F. acknowledges the support by the project INCT-FNA (464898/ 2014-5).
[1] J. Bartels, E. Gotsman, E. Levin, M. Lublinsky, and U. Maor, Phys. Lett. B 556, 114 (2003).

[2] F. Carvalho, F. O. Duraes, V. P. Goncalves, and F. S. Navarra, Mod. Phys. Lett. A 23, 2847 (2008).

[3] C. A. Arguelles, F. Halzen, L. Wille, M. Kroll, and M. H. Reno, Phys. Rev. D 92, 074040 (2015).

[4] F. Gelis, E. Iancu, J. Jalilian-Marian, and R. Venugopalan, Annu. Rev. Nucl. Part. Sci. 60, 463 (2010).

[5] H. Weigert, Prog. Part. Nucl. Phys. 55, 461 (2005).

[6] J. Jalilian-Marian and Y. V. Kovchegov, Prog. Part. Nucl. Phys. 56, 104 (2006).

[7] A. C. Caldwell and M. S. Soares, Nucl. Phys. A696, 125 (2001).

[8] H. Kowalski and D. Teaney, Phys. Rev. D 68, 114005 (2003).

[9] J. R. Forshaw, R. Sandapen, and G. Shaw, Phys. Rev. D 69, 094013 (2004).

[10] C. Marquet, R. B. Peschanski, and G. Soyez, Phys. Rev. D 76, 034011 (2007).

[11] H. Kowalski, L. Motyka, and G. Watt, Phys. Rev. D 74, 074016 (2006).

[12] N. Armesto and A. H. Rezaeian, Phys. Rev. D 90, 054003 (2014).

[13] J. R. Forshaw, R. Sandapen, and G. Shaw, J. High Energy Phys. 11 (2006) 025.

[14] K. J. Golec-Biernat and M. Wusthoff, Phys. Rev. D 60, 114023 (1999).

[15] N. N. Nikolaev and B. G. Zakharov, Z. Phys. C 64, 631 (1994).

[16] N. N. Nikolaev, B. G. Zakharov, and V. R. Zoller, JETP Lett. 59, 6 (1994), http://www.jetpletters.ac.ru/ps/1295/article_ 19555.pdf.

[17] J. R. Cudell and O. V. Selyugin, Phys. Lett. B 662, 417 (2008).

[18] J. R. Cudell, E. Predazzi, and O. V. Selyugin, Phys. Rev. D 79, 034033 (2009).

[19] Z. Chen and A. H. Mueller, Nucl. Phys. B451, 579 (1995).

[20] A. M. Stasto, K. J. Golec-Biernat, and J. Kwiecinski, Phys. Rev. Lett. 86, 596 (2001).

[21] B. Z. Kopeliovich, J. Nemchick, N. N. Nikolaev, and B. G. Zakharov, Phys. Lett. B 309, 179 (1993).

[22] , Phys. Part. Nucl. 37, 1 (2006).

[23] N. N. Nikolaev and B. G. Zakharov, Z. Phys. C 49, 607 (1991).

[24] N. N. Nikolaev and B. G. Zakharov, Z. Phys. C 53, 331 (1992).

[25] A. H. Mueller, Nucl. Phys. B415, 373 (1994).
[26] A. H. Mueller and B. Patel, Nucl. Phys. B425, 471 (1994).

[27] K. J. Golec-Biernat and M. Wusthoff, Phys. Rev. D 59, 014017 (1998).

[28] K. Golec-Biernat and S. Sapeta, J. High Energy Phys. 03 (2018) 102.

[29] M. Tanabashi et al. (Particle Data Group), Phys. Rev. D 98, 030001 (2018).

[30] F. Nemes, Recent results from 2.76 and $13 \mathrm{TeV} p+p$ collisions from the TOTEM experiment at CERN LHC, in Zimányi School 2018, Budapest, Hungary (2019).

[31] G. Antchev et al. (TOTEM Collaboration), Eur. Phys. J. C 79, 103 (2019).

[32] G. Antchev et al. (TOTEM Collaboration), Eur. Phys. J. C 79, 785 (2019).

[33] G. Antchev et al. (TOTEM Collaboration), Eur. Phys. J. C 76, 661 (2016).

[34] M. Aaboud et al. (ATLAS Collaboration), Phys. Lett. B 761, 158 (2016).

[35] G. Aad et al. (ATLAS Collaboration), Nucl. Phys. B889, 486 (2014).

[36] G. Antchev et al. (TOTEM Collaboration), Nucl. Phys. B899, 527 (2015).

[37] G. Antchev et al. (TOTEM Collaboration), Europhys. Lett. 101, 21004 (2013).

[38] L. Motyka, Acta Phys. Pol. B 34, 3069 (2003), https://www .actaphys.uj.edu.pl/fulltext?series $=$ Reg \&vol=34\&page $=3069$.

[39] R. Fiore, P. V. Sasorov, and V. R. Zoller, JETP Lett. 96, 687 (2013).

[40] N. N. Nikolaev, G. Piller, and B. G. Zakharov, Z. Phys. A 354, 99 (1996).

[41] B. Z. Kopeliovich and A. V. Tarasov, Nucl. Phys. A710, 180 (2002).

[42] N. N. Nikolaev, W. Schafer, and B. G. Zakharov, Phys. Rev. D 72, 114018 (2005).

[43] D. A. Fagundes, E. G. S. Luna, M. J. Menon, and A. A. Natale, Nucl. Phys. A886, 48 (2012).

[44] C. A. S. Bahia, M. Broilo, and E. G. S. Luna, Phys. Rev. D 92, 074039 (2015).

[45] M. Broilo, D. A. Fagundes, E. G. S. Luna, and M. J. Menon, Phys. Lett. B 799, 135047 (2019).

[46] A. I. Shoshi, F. D. Steffen, and H. J. Pirner, Nucl. Phys. A709, 131 (2002).

[47] C. Flensburg, G. Gustafson, and L. Lonnblad, Eur. Phys. J. C 60, 233 (2009).

[48] H. G. Dosch, E. Ferreira, and A. Krämer, Phys. Rev. D 50, 1992 (1994). 
[49] N. N. Nikolaev, J. Speth, and V. R. Zoller, Phys. Lett. B 473, 157 (2000).

[50] P. Abreu et al. (Pierre Auger Collaboration), Phys. Rev. Lett. 109, 062002 (2012).

[51] R. U. Abbasi et al. (Telescope Array Collaboration), Phys. Rev. D 92, 032007 (2015).

[52] A. Donnachie and H. G. Dosch, Phys. Rev. D 65, 014019 (2001).

[53] A. H. Rezaeian and I. Schmidt, Phys. Rev. D 88, 074016 (2013).

[54] F. G. Ben, M. V. T. Machado, and W. K. Sauter, Phys. Rev. D 96, 054015 (2017).

[55] R. Brun and F. Rademakers, Nucl. Instrum. Methods Phys. Res., Sect. A 389, 81 (1997).

[56] R. A. Ryutin, Eur. Phys. J. C 77, 114 (2017); 77, 843(E) (2017).

[57] V. A. Khoze, A. D. Martin, and M. G. Ryskin, Phys. Rev. D 96, 034018 (2017).

[58] M. M. Block, L. Durand, P. Ha, and D. W. McKay, Phys. Rev. D 88, 014006 (2013).

[59] M. M. Block, L. Durand, P. Ha, and D. W. McKay, Phys. Rev. D 88, 013003 (2013).

[60] M. Broilo, E. G. S. Luna, and M. J. Menon, Phys. Rev. D 98, 074006 (2018).

[61] J. R. Cudell, V. V. Ezhela, P. Gauron, K. Kang, Yu. V. Kuyanov, S. B. Lugovsky, E. Martynov, B. Nicolescu, E. A. Razuvaev, and N. P. Tkachenko (COMPETE Collaboration), Phys. Rev. Lett. 89, 201801 (2002).

[62] J. R. Cudell, V. V. Ezhela, P. Gauron, K. Kang, Yu. V. Kuyanov, S. B. Lugovsky, B. Nicolescu, and N.P. Tkachenko, Phys. Rev. D 65, 074024 (2002).

[63] J. R. Cudell et al., Forward observables at RHIC, the Tevatron run II and the LHC, in Diffraction 2002: Interpretation of the New Diffractive Phenomena in Quantum Chromodynamics and in the S Matrix Theory. Proceedings, NATO Advanced Research Workshop, Alushta, Ukraine (Kluwer Academic, Dordrecht, Netherlands, 2003) p.. 325.

[64] O. Adriani et al. (LHCf Collaboration), Phys. Lett. B 750, 360 (2015).

[65] R. Fiore, N. N. Nikolaev, and V. R. Zoller, JETP Lett. 99, 363 (2014).
[66] G. Antchev et al. (TOTEM Collaboration), arXiv: 1812.08610 .

[67] G. Antchev et al. (TOTEM Collaboration), Europhys. Lett. 101, 21002 (2013).

[68] G. Antchev et al. (TOTEM Collaboration), Eur. Phys. J. C 79, 861 (2019).

[69] B. Z. Kopeliovich, I. K. Potashnikova, and I. Schmidt, Braz. J. Phys. 37, 473 (2007).

[70] M. L. Good and W. D. Walker, Phys. Rev. 120, 1857 (1960).

[71] J. C. M. Armitage et al., Nucl. Phys. B194, 365 (1982).

[72] D. Bernard et al. (UA4 Collaboration), Phys. Lett. B 186, 227 (1987).

[73] G. J. Alner et al. (UA5 Collaboration), Phys. Rep. 154, 247 (1987).

[74] N. A. Amos et al. (E710 Collaboration), Phys. Lett. B 301, 313 (1993).

[75] F. Abe et al. (CDF Collaboration), Phys. Rev. D 50, 5535 (1994).

[76] B. Abelev et al. (ALICE Collaboration), Eur. Phys. J. C 73, 2456 (2013).

[77] M. Berretti (TOTEM Collaboration), Proc. Sci., DIS2013 (2013) 066.

[78] R. Ciesielski (CMS Collaboration), Proc. Sci., DIS2013 (2013) 091.

[79] C. Flensburg and G. Gustafson, J. High Energy Phys. 10 (2010) 014.

[80] U. Maor, Soft scattering re-visited, in Elastic and Diffractive Scattering. Proceedings, 13th International Conference, Blois Workshop (CERN, Geneva, 2009), pp. 99-106.

[81] U. Maor, AIP Conf. Proc. 1105, 248 (2009).

[82] M. Genovese, N. N. Nikolaev, and B. G. Zakharov, Zh. Eksp. Teor. Fiz. 108, 1155 (1995), http://www.jetp.ac.ru/ cgi-bin/dn/e_081_04_0633.pdf. [J. Exp. Theor. Phys. 81, 633 (1995)].

[83] Y. V. Kovchegov and K. Tuchin, Phys. Rev. D 65, 074026 (2002).

[84] G. Sampaio dos Santos and M. V. T. Machado, Eur. Phys. J. A 50, 166 (2014).

[85] V. P. Goncalves, R. P. da Silva, and P. V. R. G. Silva, Phys. Rev. D 100, 014019 (2019). 